Running Head: GLASS CURVATURE INFLUENCES THE FLAVOUR OF BEER

\title{
Glass shape influences the flavour of beer
}

Adrian Mirabito, Markus Oliphant, George Van Doorn ${ }^{1}$, Shaun Watson ${ }^{1}$, \& Charles Spence ${ }^{2}$

${ }^{1}$ School of Health Sciences and Psychology, Federation University Australia, Victoria, 3842, Australia: george.vandoorn@federation.edu.au

${ }^{2}$ Crossmodal Research Laboratory, Department of Experimental Psychology, University of Oxford, Oxford, UK

\begin{abstract}
It is often said that our perception of wine varies as a function of the receptacle in which it is presented. Indeed, glassware has been the subject of extensive study in this category. By contrast, the impact of glassware on the perception of beer has been largely ignored in the field of sensory science research. The current study was specifically designed to investigate the influence of the shape (specifically side curvature) of the glass on people's perception of beer. Fifty-three Australian participants rated (on 10-point Likert scales) a beer presented in one of two glasses. The beer was perceived as being fruitier and more intense when served in a curvedsided glass. Given previous research showing that people match fruitiness with curvature (rather than straightness/angularity), these results fit within the existing literature on crossmodal correspondences between shape and taste properties.
\end{abstract}

KEYWORDS: Beer, Glass shape, Taste, Flavour, Crossmodal correspondences 


\section{Introduction}

Beer is the most popular alcoholic beverage worldwide by volume, with global production steadily increasing from approximately 1.3 billion hectolitres in 1998 to almost two billion hectolitres in 2013 (Statista, 2016). Given its growing global popularity, it becomes increasingly important to try and understand how the taste/flavour of beer is perceived and, more importantly, the factors that might enhance the perception of its various sensory qualities (see Wells, 2015). Although a few studies have been conducted on the productextrinsic factors that influence people's perception of the taste/flavour of beer (e.g., Carvalho, Wang, de Causmaecker, Steenhaut, Van Ee, \& Spence, 2016; Carvalho, Wang, Van Ee, \& Spence, 2016; Sester, Deroy, Sutan, Galia, Desmarchelier, Valentin, \& Dacremont, 2013), this most popular of alcoholic drinks seems to have been somewhat neglected by researchers. The experiment reported here was designed to explore the influence of the shape of the beer glass on the perception of the characteristics of the contents.

\subsection{Shape-taste/flavour correspondences}

A product's shape, not to mention the shape of its container, associated logo, and any relevant typeface, have been shown to influence many aspects of consumer behaviour (Velasco, Woods, Hyndman, \& Spence, 2015; see Spence, 2012, for a review), and any shapes that are present on, or near, a food or beverage can influence their perceived qualities (Gal, Wheeler, \& Shiv, 2007). In general, people tend to prefer rounded shapes (e.g., circles) over more angular shapes (e.g., triangles or stars; Bar \& Neta, 2006; Gómez-Puerto, Munar, \& Nadal, 2015; Silvia \& Barona, 2009), and this preference has been observed to extend to food. For instance, in early research, Cheskin (1957) drew attention to the impact of background shapes on people's perception of different products by placing identical products (e.g., crackers) in two different packages, one adorned with triangles, the other with circles. Eighty-percent of the participants preferred the product in the package with circles, often suggesting, when asked, that this product was of 'better quality' (see Westerman et al., 2012, for similar results in relation to product packaging).

Shape has also been shown to influence the drinking experience. For instance, Attwood et al. (2012) reported that participants drank an alcoholic beverage (i.e., a lager beer) more slowly from a straight-sided glass than from a glass with curved sides. Interestingly, consumption speed was not affected when the glasses were only half-filled, or when non-alcoholic beverages were presented instead. Similarly, the shape of the glass was found to influence how much of 
the alcoholic drink was consumed but, once again, did not influence how much of a nonalcoholic beverage was consumed. Parenthetically, Stafford, Fernandes, and Agobiani (2012) have also demonstrated that exposure to product-extrinsic cues (i.e., attributes that, should they be changed, will not change the product or how it functions; specifically, the presence or absence of music in Stafford et al.’s study) altered the taste of alcohol which, in turn, led to increased alcohol consumption. Elsewhere, Stafford and Dodd (2013) demonstrated that the presence of music influenced consumption speed.

It is interesting that both music and shape influence alcohol consumption, and this work may be important with regard to sensorially 'nudging' health-related behaviours. One possible interpretation of the finding that the shape of a glass influenced the amount of alcohol consumed is that a crossmodal correspondence exists because glasses with curved sides along the vertical dimension have been paired with alcoholic beverages repeatedly in the marketplace, and thus a sensory feature in vision is paired with a sensory attribute in taste (see Ernst, 2007; Schifferstein, 2009; Wan, Woods, Jacquot, Knoeferle, Kikutani, \& Spence, 2016, for other examples). Interestingly, consistently and congruently paired stimuli promote processing fluency (the term 'processing fluency' refers to the ease with which a person can process a stimulus ensemble; Reber, Schwartz, \& Winkielman, 2004) which, in turn, might have motivated the increased consumption of alcohol from these specific product-congruent receptacles.

Researchers have also demonstrated that people generally enjoy drinking beverages more when the receptacle is deemed to be consistent with the contents (Cavazzana, Larsson, Hoffmann, Hummel, \& Haehner, 2017; Schifferstein, 2009; Spence \& Wan, 2015). Relevant to this point, Wan, Zhou, Woods, and Spence (2015) found that people reported being willing to pay more for a beer when it was presented in a dimpled beer mug, relative to another glass (e.g., a highball or wine glass). They also found that participants preferred the beer when it was presented in the dimpled beer mug, relative to other glass types. Given this information, glass shape seems to play an important role in the perception of beer.

The influence of glass shape on the perceived aroma of wine has been studied extensively. For example, Delwiche and Pelchat (2002) have demonstrated that glass type influences people's ratings of wine aroma. Similarly, Vilanova, Vidal, and Cortez (2008) found that wine experts' ratings along dimensions such as aroma, taste, and quality, were influenced by glass shape. Several authors contend that the physical properties of glasses play a role in our ability to perceive the aroma released from the beverage’s surface. For example, Cliff (2001) suggested 
that larger openings allow aromas to escape prior to evaluation, while others (e.g., Spence, 2011, 2016) have noted that a small-diameter glass allows fewer odour molecules to released from the surface of the liquid. That said, and consistent with the argument outlined above, it may also be that certain glass shapes have been paired with wine consistently enough to establish an association between the two - think, for example, of the Bordeaux glass or champagne flute. This cognitive association may, in turn, influence aroma perception just as much as any more fundamental associations between flavour and shape properties.

Interestingly, there is a separate body of research assessing the aromas associated with carbonated beverages. For example, Liger-Belair $(2003,2005)$ studied the factors influencing the perceived aroma of champagne. Of relevance here, Liger-Belair, Bourget, Pron, Polidori, and Cilindre (2012) found that the shape of champagne glasses has the potential to influence the perception of aroma. Specifically, the commonly-used flute glass concentrates $\mathrm{CO}_{2}$ which might interfere with a consumer's ability to detect specific aromas. On the other hand, coupe style glasses dilute $\mathrm{CO}_{2}$ which, in turn, will dilute perceived aromas. As such, perhaps a glass that melds features of these two glass types should be used when drinking champagne. Elsewhere, Cavazzana et al. (2017) assessed the influence of glass shape on the perceived taste and aroma of a cola drink. The cola was rated as more pleasant, and more intense, when presented in a receptable that was deemed consistent with the contents (i.e., a typical CocaCola ${ }^{\circledR}$ glass) than when it was presented in a receptacle that was deemed inconsistent with the contents (e.g., a plastic bottle). As noted by the authors, though, weight differences may have contributed to perceived differences in the drink’s sensory qualities as well (cf. Gatti, Spence, \& Bordegoni, 2014).

Although associations between shape and taste have been explored in a range of food and beverage products, the correspondence between shape and the perception of beer remains essentially unknown. Furthermore, the consistent, bitter character and the different bitter/sweet combinations of beers make it an intriguing topic for empirical research.

\subsection{Aims and hypotheses}

Given that, in the setting of a bar, beer is often served in a glass, the primary aim of the present study was to explore the impact of the shape of glasses on people's perception of beer. Similar to previous work (e.g., Attwood et al., 2012; Wan et al., 2015), we explored the influence of the shape, as either curved or straight, of the glass the beverage was served in. 
It is known that people tend to form associations between the physical properties of a stimulus (e.g., its shape) and particular tastes. For example, people typically associate round shapes with sweetness (see Deroy \& Valentin, 2011; Spence, 2012). As such, we expected that people would rate the beer presented in glasses with curved sides along the vertical dimension as tasting somewhat sweeter than the beer presented in glasses with straight sides on the vertical dimension. Similarly, people sometimes confuse sweetness and fruitiness (cf. Harrar, Smith, Deroy, \& Spence, 2013) and, as such, it was expected that people would rate the beer presented in glasses with curved sides along the vertical dimension as being fruitier than the beer presented in glasses with straight sides on the vertical dimension. As mentioned already, people generally enjoy beverages more when they are served in a receptacle that is deemed to be congruent with the contents (Schifferstein, 2009; Spence \& Wan, 2015) than when the beverage is presented in a receptacle that is deemed incongruent. Furthermore, it has recently been shown that people prefer, and are willing to pay more for, a beer when it is served in a glass 'beer' mug compared to when it is served in a non-standard container (Wan et al., 2015). It is currently common practice to serve beer in curved glasses in pubs across Australia. We, therefore, expected people to rate these glasses as containing beer that was more pleasant, and we hypothesised that participants would be willing to pay more for the beer served in the glass with curved sides.

\section{Methods}

\subsection{Participants}

Fifty-three participants ( $M=24.91$ years, $S D=8.39$ years; range 18 - 64 years $)$ took part in the experiment. The sample comprised 28 males and 25 females. The participants were asked to "best describe" their beer drinking behaviour. Approximatley half of the participants indicated that they either 'never' drank beer or 'only sometimes' drank beer. The participants were recruited from either a Psychology participant pool, or were members of the student body, staff, or general public who were on-campus at a regional university. Participants recruited from the participant pool received course credit in order to fulfil the requirements of an introductory psychology course they were enrolled in. All other participants took part in the experiment voluntarily. The experiment was approved by the university's ethics committee, and conformed to the ethical standards for conducting research established by the American Psychological Association.

\subsection{Materials}




\subsubsection{Glasses}

The two glasses chosen for this experiment were both transparent and colourless (see Figure 1). One was a more traditional beer glass with curved sides, as typically found in Australian pubs, while the other had straight sides. Metal washers of different sizes and weights were affixed to the bottom of each glass in order to minimise any weight discrepancies between the glasses (see Figure 2). The relative weight discrepancies between the two glass types ranged between 42-69g, which was reduced to no more than 1-3g after attaching washers to the bottom of each glass (see Gatti et al., 2014; Spence \& Piqueras-Fiszman, 2011, for evidence of the influence of weight on olfactory perception). Furthermore, attaching washers to the bottom of each glass (instead of just the lighter, straight-sided glasses) removed the possibility that washers on only one of the two glasses would influence participant behaviour (e.g., taste ratings of beer across glass shapes). It was not possible to eliminate the height discrepancy between the glasses. However, curved-sided and straight-sided glasses were never shown at the same time and, as such, it is unlikely that such minor differences in glass height would have influenced participants’ ratings.

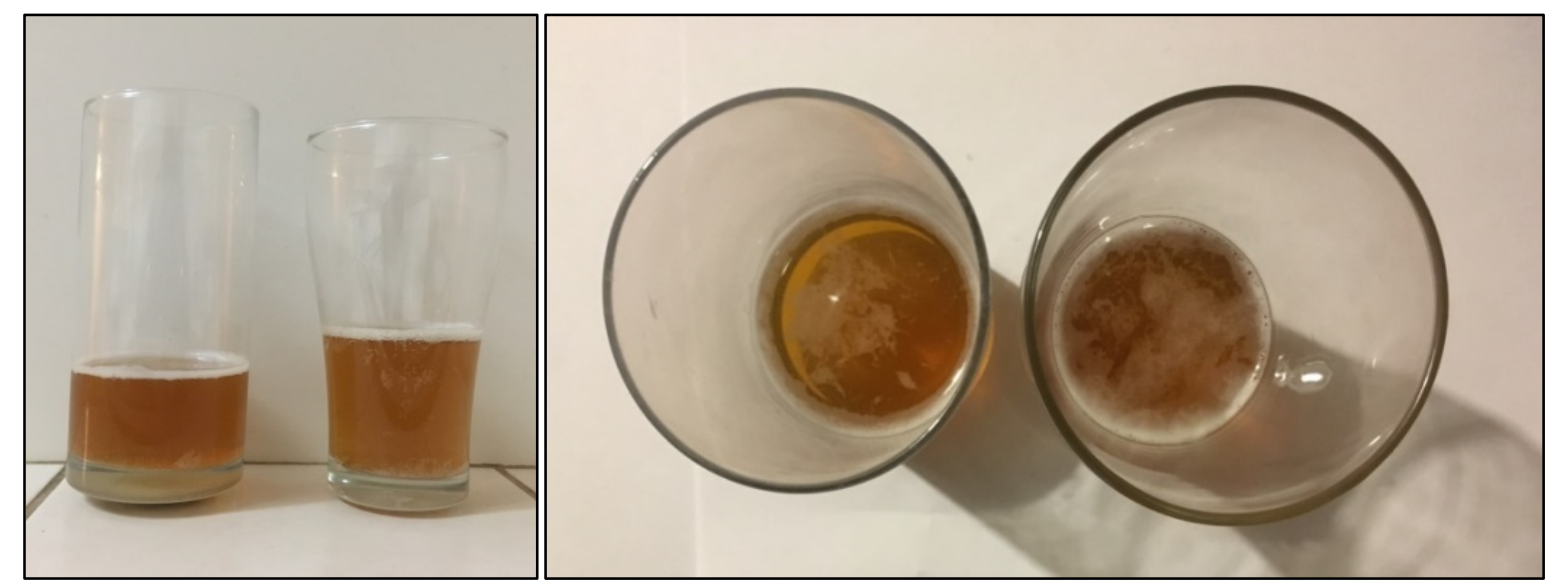

Figure 1. Panel A: Side-view of the straight and curved glasses and 100mL Yenda Pale Ale in each glass. Panel B: Bird's-eye view of the glasses with 100mL Yenda Pale Ale in each glass. 


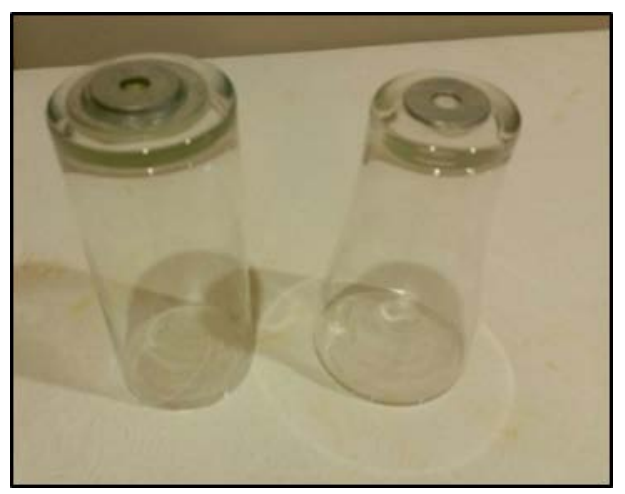

Figure 2. The straight and curved glasses with washers attached.

\subsubsection{Beer}

Yenda Pale Ale was provided to participants in all experimental conditions. This craft beer contained $4.5 \%$ alcohol per $330 \mathrm{~mL}$ bottle. The beer was refrigerated between 4 and $6^{\circ} \mathrm{C}$ prior to the experiment. Manufactured by the Australian Beer Company, the beer is described as a fruity, crisp, biscuity, malt flavoured beer of medium intensity, as well as being of medium hop and a medium amber intensity (Australian Beer Company, 2015). This beer was selected over other more popular and highly advertised beers in an attempt to avoid participants recognising the beer’s taste (though see Allison \& Uhl, 1964).

\subsection{Design}

A repeated-measures experimental design was used that included the within-participants factors of 'glass shape' (curved, straight). The dependent variables (DVs) are outlined in Table 1. It is known that certain terms commonly used to describe the attributes of beer are not well understood by non-experts (cf. Peron \& Allen, 1988). As such, the present study used four descriptors (intensity, sweetness, bitterness, and fruitiness) to assess the taste characteristics of beer. In addition to these facets of flavour, the overall pleasantness of the taste of the beer was evaluated. Further descriptors that are useful when assessing beer have been provided by research assessing the perceived characteristics of other types of alcohol. For example, and as mentioned above, research into wine has evaluated the effects of glass shape on perceived aroma (Vilanova et al., 2008). While there is little research examining aroma perception in relation to beers presented in different glass shapes, it seemed logical to extend the work done with wine to another alcoholic beverage. 
Table 1. Dependent Variables, Questions Asked to Assess Each, and Scale Anchors Used to Rate Beer Attributes

\begin{tabular}{lll}
\hline Dependent variable & Question asked & Scale anchors (left to right) \\
\hline Aroma & $\begin{array}{l}\text { How pleasant would you rate the aroma of the } \\
\text { beer? }\end{array}$ & $\begin{array}{l}\text { Extremely unpleasant; Extremely } \\
\text { pleasant }\end{array}$ \\
Bitterness & How bitter would you rate the taste of the beer? & Not bitter at all; Extremely bitter \\
Fruitiness & How fruity would you rate the taste of the beer? & Not fruity at all; Extremely fruity \\
Intensity & How intense would you rate the taste of the beer? & Extremely mild; Extremely \\
& & intense \\
Taste pleasantness & $\begin{array}{l}\text { How pleasant would you rate the taste of the } \\
\text { beer? }\end{array}$ & $\begin{array}{l}\text { Not pleasant at all; Extremely } \\
\text { pleasant }\end{array}$ \\
Sweetness & How sweet would you rate the taste of the beer? & Not sweet at all; Extremely sweet \\
Willingness to pay & $\begin{array}{l}\text { How much would you be willing to pay for a full } \\
\text { glass of this beer? }\end{array}$ & $\begin{array}{l}\text { Open-ended response (Australian } \\
\text { dollars) }\end{array}$ \\
\hline
\end{tabular}

\subsection{Procedure}

Prior to entering the experimental room, the participants were informed that they would be sampling four different types of beer. The participants were then taken into the experimental room individually and asked to either sit or stand, and familiarise themselves with the questionnaire provided by the experimenter. It should be noted that while posture was included as an exploratory variable in this study, no impact of this factor was seen on the results and, to keep the results section simple, it will not be mentioned further. During this time, and out-ofsight of the participants, the experimenter poured $100 \mathrm{~mL}$ of Yenda Pale Ale into each of the four glasses (i.e., 2 [posture] x 2 [glass type] = 4 beers). Care was taken to ensure that the beer did not froth when poured.

The experimenter brought one glass of beer and a glass of water to the participant, and instructed them to fill-in the questionnaire between sips of beer. This instruction was given in order to improve the accuracy of the participants' ratings. The participants were also instructed to cleanse their mouths with water after completing each condition. The participants were informed that consumption of the entire amount of beer provided in each glass was not mandatory, and they were instructed to call out to the experimenter when they had completed 
the condition, or if they had any questions. The experimenter waited in the cool room during each condition. After completing a condition, the experimenter collected the completed questionnaire and used beer glass, and provided each participant with the next glass of beer and a new questionnaire. The order of the items on each questionnaire was randomised for each condition.

Once the participant had completed the experimental conditions, the experimenter debriefed them. The latter also advised the participants to refrain from driving for 1-2 hours and reminded each participant that the legal blood alcohol concentration for provisional drivers (in Victoria, Australia) is $0.00 \%$ (i.e., 0.00 grams of alcohol in every 100 millilitres of blood; VicRoads, 2016). The used glasses were washed thoroughly under hot water and any half-empty bottles of beer were re-sealed and placed back into the refrigerator. All conditions were counterbalanced across participants, although obviously not perfectly given the odd number of participants, in an attempt to control for order effects.

\subsection{Analyses}

Seven repeated-measures Analyses of Variance (ANOVAs) were conducted that were identical in terms of design except for their dependent variable (Aroma, Bitterness, Fruitiness, Intensity, Pleasantness of taste, Sweetness, and Willingness-to-pay); the dependent variable was the rating on a 10-point Likert scale. Each ANOVA consisted of the repeated-measures factor of 'glass shape' (straight vs. curved).

\section{Results and Discussion}

The data from one male participant was removed prior to analysis as he failed to comply with the instructions. His demographic details were not included in the descriptive statistics presented above (i.e., in the 'Participants' section). Although the dataset contained missing values due to participant non-response, this value did not exceed a cut-off of $5 \%$ for any item (Field, 2005). As such, missing data were not replaced. In all, seven data points were missing. Little's MCAR test was run to assess whether data were missing at random. The test was not significant $\chi^{2}=(169, \mathrm{~N}=53)=159.69, p=.679$, and thus there was no identifiable pattern to the missing data.

With 'fruitiness' as the DV, the main effect of 'glass shape' was significant $[F(1,51)=4.81, p$ $\left.=.033, \eta^{2}{ }_{p}=.086\right]$. The beer associated with the glass with the curved sides was perceived to be fruitier than the beer served in the glass with the straight sides. This result may reflect the fact that participants associate the curved shape of the sides of the glass with the rounded shape 
of many fruits. Research has shown that people develop associations between seemingly unrelated stimulus features, and these associations can play a role in how we perceive the taste of many foods and drinks (see Crisinel, Jones, \& Spence, 2012; Crisinel \& Spence, 2010; Velasco et al., 2015).

Lastly, when 'intensity' was used as the DV, the main effect of 'glass shape' $[F(1,51)=5.90$, $\left.p=.019, \eta_{\mathrm{p}}^{2}=.104\right]$ was significant. The beer in the glass with the curved sides was perceived as being more intense than was the beer served in the glass with the straight sides. The full report of the analyses is given in Table 2. Here, we have argued for learned associations over innate correspondences. Future research could explore the outcomes of serving novel drinks in curved-sided and straight-edged glasses. It is difficult to generalise whether or not the fruitiness and intensity findings would hold for novel beverages. That said, research by Wan et al. (2015) found that people were willing-to-pay more for alcoholic beverages presented in glasses deemed consistent with the drink (a learned association) than they were for novel (unlearned) pairings.

Table 2: Results of Separate Repeated-Measures ANOVAs for each of the Dependent Variables

\begin{tabular}{|c|c|c|c|c|c|c|c|}
\hline & Curved & lass & Straigh & lass & & & \\
\hline Attribute & $M$ & $S E$ & $M$ & $S E$ & $F$ & Sig. & $\begin{array}{c}\text { Partial Eta } \\
\text { Squared }\end{array}$ \\
\hline Aroma & 5.79 & .23 & 5.69 & .26 & 0.376 & .542 & 0.007 \\
\hline Bitterness & 5.71 & .25 & 5.46 & .25 & 1.666 & .203 & 0.032 \\
\hline Fruitiness & 3.91 & .29 & 3.43 & .26 & 4.813 & $.033 *$ & 0.086 \\
\hline Intensity & 6.25 & .18 & 5.86 & .19 & 5.900 & $.019 *$ & 0.104 \\
\hline Pleasantness & 5.98 & .27 & 6.09 & .26 & 0.232 & .632 & 0.005 \\
\hline Sweetness & 3.97 & .26 & 4.16 & .26 & 0.936 & .338 & 0.018 \\
\hline Willingness to Pay & 5.27 & .21 & 5.36 & .22 & 0.408 & .526 & 0.008 \\
\hline
\end{tabular}

$*=p<.05$

\section{Conclusion}

The main issue explored in the present study was whether people's perception of a glass of beer would be influenced by changes in the shape of the glass itself. The results revealed that 'glass shape' influenced the perceived intensity ( 4\%) and fruitiness $(\sim 5 \%)$ of the beer. Therefore, based on the present results, those bar owners/workers, restauranteurs, and glass manufacturers wanting to manipulate people’s perception of beer should carefully consider the 
curvature of the glasses they use/produce, as these features will likely affect perceived intensity and fruitiness. It is worth mentioning that there is a heavy emphasis on the influence of glass shape on the taste of wine in the literature. The work presented here suggests that glassware, as it pertains to other, non-grape-based beverages, should be explored. Furthermore, given our findings, one might also wonder just how much sense it makes to conduct blind taste tests without knowing something about the glassware from which the drink is normally consumed. 


\section{REFERENCES}

Allison, R. I., \& Uhl, K. P. (1964). Influence of beer brand identification on taste perception. Journal of Marketing Research, 1, 36-39.

Attwood, A. S., Scott-Samuel, N. E., Stothart, G., \& Munafò, M. R. (2012). Glass shape influences consumption rate for alcoholic beverages. PLoS ONE, 7(8):e43007. doi: 10.1371/journal.pone.0043007

Australian Beer Company (2015). Yenda beer. Retrieved from https://australianbeerco.com.au/our-brews/beers/

Bar, M., \& Neta, M. (2006). Humans prefer curved visual objects. Psychological Science, 17(8), 645-648.

Carvalho, F. R., Wang, Q. (J.), de Causmaecker, B., Steenhaut, K., Van Ee, R., \& Spence, C. (2016). Tune that beer! Listening for the pitch of beer. Beverages, 2(4):31.

Carvalho, F. R., Wang, Q. (J.), Van Ee, R., \& Spence, C. (2016). The influence of soundscapes on the perception and evaluation of beers. Food Quality and Preference, 52, 32-41.

Cavazzana, A., Larsson, M., Hoffmann, E., Hummel, T., \& Haehner, A. (2017). The vessel's shape influences the smell and taste of cola. Food Quality and Preference, 59, 8-13.

Cheskin, L. (1957). How to predict what people will buy. New York, NY: Liveright.

Cliff, M. (2001). The influence of wine glass shape on perceived aroma and colour intensity in wines. Journal of Wine Research, 12, 39-46.

Crisinel, A.-S., Jones, S., \& Spence, C. (2012). 'The sweet taste of maluma': Crossmodal associations between taste and words. Chemosensory Perception, 5(3-4), 266-273.

Crisinel, A.-S., \& Spence, C. (2010). A sweet sound? Food names reveal implicit associations between taste and pitch. Perception, 39(3), 417-425. doi: 10.1068/p6574.

Delwiche, J. F., \& Pelchat, M. L. (2002). Influence of glass shape on wine aroma. Journal of Sensory Studies, 17(1), 19-28. doi: 10.1111/j.1745-459X.2002.tb00329

Deroy, O., \& Valentin, D. (2011). Tasting liquid shapes: Investigating the sensory bias of crossmodal correspondences. Chemosensory Perception, 4, 80-90.

Ernst, M. O. (2007). Learning to integrate arbitrary signals from vision and touch. Journal of Vision, 7(5), 1-14. doi:10.1167/7.5.7

Field, A. (2005). Discovering statistics using SPSS ( $2^{\text {nd }}$ Ed.). London, UK: Sage.

Gal, D., Wheeler, S. C., \& Shiv, B. (2007, unpublished manuscript). Cross-modal influences on gustatory perception. Available at SSRN: http://ssrn.com/abstract=1030197

Gatti, E., Spence, C., \& Bordegoni, M. (2014). Investigating the influence of colour, weight, \& fragrance intensity on the perception of liquid bath soap. Food Quality \& Preference, 31, 56-64.

Gómez-Puerto, G., Munar, E., \& Nadal, M. (2015). Preference for curvature: A historical and conceptual framework. Frontiers in Human Neuroscience, 9:712.

Harrar, V., Smith, B., Deroy, O., \& Spence, C. (2013). Grape expectations: How the proportion of white grape in Champagne affects the ratings of experts and social drinkers in a blind tasting. Flavour, 2:25. 
Liger-Belair, G. (2003). The science of bubbly. Scientific American, 288(1), 80-85.

Liger-Belair, G. (2005). The physics and chemistry behind the bubbling properties of champagne and sparkling wines: A state-of-the-art review. Journal of Agricultural and Food Chemistry, 53, 2788-2802.

Liger-Belair, G., Bourget, M., Pron, H., Polidori, G., \& Cilindre, C. (2012). Monitoring gaseous $\mathrm{CO}_{2}$ and ethanol above champagne glasses: Flute versus coupe, and the role of temperature. PLoS ONE, 7(2): e30628. doi:10.1371/journal.pone.0030628

Peron, R. M., \& Allen, G. L. (1988). Attempts to train novices for beer flavour discrimination: A matter of taste. The Journal of General Psychology, 115(4), 403-418. doi: 10.1080/00221309.1988.9710577.

Reber, R., Schwarz, N., \& Winkielman, P. (2004). Processing fluency and aesthetic pleasure: Is beauty in the perceiver's processing experience? Personality and Social Psychology Review, 8, 364-382.

Schifferstein, H. N. J. (2009). The drinking experience: Cup or content? Food Quality and Preference, 20, 268-276.

Sester, C., Deroy, O., Sutan, A., Galia, F., Desmarchelier, J.-F., Valentin, D., \& Dacremont, C. (2013). "Having a drink in a bar": An immersive approach to explore the effects of context on beverage choice. Food Quality and Preference, 28, 23-31.

Silvia, P. J., \& Barona, C. M. (2009). Do people prefer curved objects? Angularity, expertise, and aesthetic preference. Empirical Studies of the Arts, 27(1), 25-42.

Spence, C. (2011). Crystal clear or gobbletigook? The World of Fine Wine, 33, 96-101.

Spence, C. (2012). Managing sensory expectations concerning products and brands: Capitalizing on the potential of sound and shape symbolism. Journal of Consumer Psychology, 22(1), 37-54.

Spence, C. (2016). Enhancing the experience through smell. Food Science and Technology, 30(2), 32-35.

Spence, C., \& Ngo, M. K. (2012). Assessing the shape symbolism of the taste, flavour, and texture of foods and beverages. Flavour, 1:12.

Spence, C., \& Piqueras-Fiszman, B. (2011). Multisensory design: Weight and multisensory product perception. In G. Hollington (Ed.), Proceedings of RightWeight2 (pp. 8-18). London, UK: Materials KTN.

Spence, C., \& Wan, X. (2015). Beverage perception and consumption: The influence of the container on the perception of the contents. Food Quality and Preference, 39, 131-140. doi: 10.1016/j.foodqual.2014.07.007

Stafford, L. D., \& Dodd, H. (2013). Music increases alcohol consumption rate in young females. Experimental and Clinical Psychopharmacology, 21(5), 408-415.

Stafford, L. D., Fernandes, M., \& Agobiani, E. (2012). Effects of noise and distraction on alcohol perception. Food Quality and Preference, 24, 218-224.

Statista (2016). Beer production worldwide from 1998 to 2014 (in billion hectoliters). Retrieved from http://www.statista.com/statistics/270275/worldwide-beer-production

Velasco, C., Woods, A. T., Deroy, O. \& Spence, C. (2015). Hedonic mediation of the crossmodal correspondence between taste and shape. Food Quality and Preference, 41, 151158. doi: 10.1016/j.foodqual.2014.11.010. 
Velasco, C., Woods, A. T., Hyndman, S., \& Spence, C. (2015). The taste of typeface. iPerception, 6(4):1-10.

Vicroads. (2016). P1 and p2 probationary license restrictions. Retrieved from https://www.vicroads.vic.gov.au/licences/your-ps/p1-and-p2-probationary-licence-restrictions

Vilanova, M., Vidal, P., \& Cortes, S. (2008). Effect of the glass shape on flavor perception of “toasted wine” from Ribeiro (NW Spain). Journal of Sensory Studies, 23(1), 114-124. doi: 10.1111/j.1745-459X.2007.00145

Wan, X., Woods, A. T., Jacquot, M., Knoeferle, K., Kikutani, M., \& Spence, C. (2016). The effects of receptable on the expected flavour of a colored beverage: Cross-cultural comparison among French, Japanese, and Norwegian consumers. Journal of Sensory Studies, 31(3), 233244.

Wan, X., Zhou, X., Woods, A. T., \& Spence, C. (2015). Influence of the glassware on the perception of alcoholic drinks. Food Quality and Preference, 44, 101-110. doi: 10.1016/j.foodqual.2015.03.018

Wells, J. (2015, July 29). Does the shape of your glass affect how a beer tastes? Retrieved from http://www.telegraph.co.uk/men/thinking-man/11765323/Does-the-shape-of-your-glassaffect-how-a-beer-tastes.html

Westerman, S. J., Gardner, P. H., Sutherland, E. J., White, T., Jordan, K., Watts, D., \& Wells, S. (2012). Product design: Preference for rounded versus angular design elements. Psychology \& Marketing, 29(8), 595-605.

Woods, A. T., Lloyd, D. M., Kuenzel, J., Poliakoff, E., Dijksterhuis, G. B., \& Thomas, A. (2011). Expected taste intensity affects response to sweet drinks in primary taste cortex. Neuroreport, 22(8), 365-369. doi:10.1097/WNR.0b013e3283469581. 